\title{
A Heuristic Research of the Designing and Evaluation of Energy Efficiency using Dynamic VM Consolidation in Green Cloud Data Centres
}

\author{
Sajitha.A.V, A.C.Subhajini
}

\begin{abstract}
Allotted computing is a blasting innovation that tenders effective assets, and smooth accessibility through web based processing. however, the growing wishes of clients for such administrations are convincing the cloud professional corporations to send huge portions of strength hungry server farms which element awful effect to the earth with the aid of the usage of plenteous Carbon Dioxide discharge. To limit control usage and strengthen the quality of service (QoS) inside the server farm assesses the strength usage in an assortment of plans in IaaS of dispensed computing situation. Dynamic Virtual Machines' Consolidation and Placement(DVMCP) is an in a position strategies for enhancing using assets and proficient power usage in Cloud DataCenters. in this exploration, we proposed a calculation, Energy Conscious Greeny Cloud Dynamic (ECGCD) set of rules that accomplishes live VM relocation that is turning off the inert has or located it to lowcontrol mode (i.e., rest or hibernation), that builds up power productivity and succesful usage of property in the dynamic hosts. The take a look at stop result confirmations with duplicate that, the proposed calculation achieves good sized diploma of lower in electricity usage in correlation with the modern-day-day VM combination calculations.
\end{abstract}

Keywords:- Energy Efficiency, VM consolidation, Host Overload Detection, Host Under load Detection, Live VM Migration

\section{INTRODUCTION}

Disbursed computing need to be taken into consideration as a sprouting system in which packages just as administrations that helps out thru a disseminated device wherein big property are gathered in massive server farms and which may be gotten to with the aid of financially, helpfully and viably via internet primarily based figuring for its large form of customers[1]. in this circumstance, strength usage is the considerable trouble. as a consequence of vitality utilization within the Cloud statistics center (CDC) for its responsibilities, for instance, stockpiling, restoration, control supply and cooling making immoderate vitality usage which again the donor of excessive operational price. presently days, EC2, Amazon, Microsoft, IBM, Google and severa massive precise corporations were conveyed CDCs for facilitating net packages. each CDC is a unified archive consists of of hundreds of bodily machines which might be displayed in numerous racks that could run jillions of virtual

Revised Manuscript Received on April 12, 2019.

Sajitha.A.V. Research Scholar, Department of Computer Applications, Noorul Islam Centre for Higher Education, TamilNadu, India (sajithaav09@gmail.com)

Dr. A.C.Subhajini, Assistant Professor,Department of Computer Applications, Noorul Islam Centre for Higher Education, TamilNadu, India. (acsubhajini@yahoo.com)
Machines. it could dissipate as heaps electricity as a little hydroelectric electricity station may also need to create [2]. The ecological effect is the $\mathrm{CO} 2$ discharge, which makes up most noteworthy portion of the inexperienced residence fuel (GHG) into the environment. it will make international Warming which is the boom of Earth's desired outdoor temperature. As said by means of the use of the herbal assets safety Council (NRDC), national, CDCs all in all wolfed 90 billion $\mathrm{kWh}$ of electrical energy in 2015, and they'll have been applied 139 billion $\mathrm{kWh}$ by using 2020.presently, CDCs expending up to a few\% of all international energy era therefore those are radiating two hundred million metric large quantities of $\mathrm{CO} 2$. As indicated with the resource of the record of eu Union, preceding 2020 to hold up the global temperature raise beneath $2{ }^{\circ} \mathrm{C}$, a lower in $\mathrm{CO} 2$ discharge volume about $15 \%-30 \%$ is basic [3].

Inexperienced figuring or green innovation determines to the eco-accommodating usage of computer systems and some different innovation associated property. because of such capability outcomes to the climate, the inexperienced allocated computing hobby has superior as a function of the green IT imaginative and prescient. green Cloud Computing (GCC) is visualized to perform now not just capable managing and suitable computational foundation usage, but moreover lessening the strength utilization. It offers a endeavor region for electricity aware $\mathrm{CDCs}[4]$.

Virtualization is a key innovation of distributed computing that gives the foundation of GCC which is going approximately as an amazing asset for putting off electricity wastefulness with the aid of using a few digital Machines (VM) in a selected bodily system(PM) by manner of live VM relocation rehearses. VM solidification is a regular near restricting the amount of strolling PMs with the useful resource of moving and mixing the VMs into the reduced range of PMs[5]. VM aggregate includes VM state of affairs (the method for choosing the most suitable host for the given $\mathrm{VM}$ ) and $\mathrm{VM}$ relocation (is the undertaking of transferring a VM beginning with one PM then onto the subsequent).

On this paper, we used to consider the live VM relocation which alludes to the development of a functioning VM separated from a PM to a few one-of-a-kind with out detaching its client, stockpiling, put together affiliation, reminiscence of the VMs which is probably gotten to from

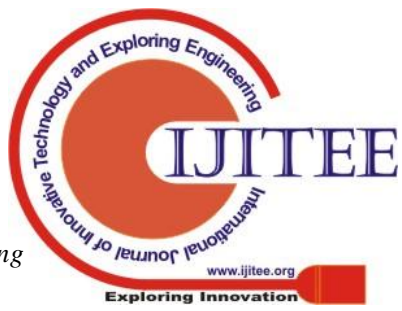




\section{A Heuristic Research Of The Designing And Evaluation Of Energy Efficiency Using Dynamic Vm Consolidation In Green Cloud Data Centres}

its particular host[6]. stay Migration is completing for project energy skillability, immoderate accessibility of bodily servers and burden adjusting in CDC. here, while the host is inactive, no VMs are provisioning, and in a while it is final down or controlled in low energy - relaxation or hibernation-kingdom. on this work, we're imparting a calculation to be precise, power conscious Greeny Cloud Dynamic set of guidelines, which aides in precise VM solidification and affiliation to stay VM relocation this is became off unused servers or placed it to sit down mode which spares energy and efficient asset use. To help the lower the quantity of dynamic PMs and development of the QoS conveyed, on each event a PM will become a hot or cold hit upon, its VMs are reassigned in a perfect machine via live VM relocation [7].

The paintings sorts as pursues: section II indicates associated works. phase III gives subtleties the framework engineering delivered on this examination. vicinity IV chats the proposed VM union device. place V examinations a presentation assessment of the proposed approach. At long ultimate, an prevent and destiny improvements are delivered in phase VI.

\section{LITERATURE REVIEW}

On this district, we painting large standpoints predicted inside the writing for project power productiveness in CDCs. within the district of dispensed computing, vitality affiliation planning and VM mixture strategies are essentially playing out the project of reaching energy skillability. there's a ton of research works are completed on VM solidification which communicates that it is the most wonderful cause at the back of the presentation and strength the board approach in CDCs. As a don't forget of first significance art work proper here had been proposed with the useful resource of Nathuji R et al. [8], for electricity the executives of CDCs by techniques for a format gadget wherein the executives of asset want to be viable thru each global and community directors. Esfandiarpoor, S. et al.[9], had been added the number one segments as an example cooling types of system, rack utilization, organize topology for the minimization of power utilization. Quanwang, W. et al.[10], had been proposed the solution for bi-purpose enhancement trouble, for instance, to restrain the VM motion price to spare electricity in keeping with dynamic VM solidification in a heterogeneous CDC. A relocation fee assessment technique and top headed appraisal technique for maximum noteworthy power sparing, a aggregate rating capacity is conceived. Fahimeh, F. et al. [11], have proposed VM union dependent on meta heuristic gadget specially Ant Colony device which achieves counterfeit ants to join VMs into a little amount of PMs in keeping with the real fantastic project at hand and ants executing their work in correspondence to make relocation of VM. The impact demonstrates the vast improvement over other various heuristic calculation through distinctive feature of the energy usage. Ibrahim, A. et al.[12] , had been created profiling and improving gadgets - The Leeds Testbed which is probably useful to recognize how the strength has been applied in a framework asset that is pivotal to assist programming planners to make strength conscious programming value determinations as some distance as energy familiarities for streamlining end result and sports. Youwei, D. et al. [13] were drew our interest approximately virtualization this is the essential aspect innovation for the strength productiveness in CDCs through the detailing of VM reserving calculation EEVS which tested to be useful to limit the whole energy usage via manner of the cloud which helped DVFS. in any case, that they'd a few inadequacies, for example, the strength punishment of circumstance changes, execution of processor and VM relocations are sincerely neglected. Zhe, H. et al. [14] have been considered VM aggregate form to be specific M-raised enhancement structure for a mechanized VM solidification procedure for allocating VMs and Servers with least framework reconfiguration. at some point of this undertaking execution productivity of server farms are completed and flexibility is advanced. Mustafa, S et al. [15] had been provided calculations named available ability and strength (ACP) and Required capability and electricity (RCP) through which a server is desired for the bottom of open CPU functionality and the power that mght be expended for that limit. while utilising the verifiable data and wisely substituting the estimations of the facts relying on changing and difficult to understand last obligations reachable, it supported to perform dynamic VM solidification. They had been established thru the exploratory very last outcomes that it recovered for the strength utilization. Nguyen $\mathrm{T}$. $\mathrm{H}$ et al.[16], were added a VM Consolidation calculation with severa usage Prediction (VMCUP-M) for enhancing strain adequacy in CDCs. It considered an collection of asset sorts and its anticipated use. whilst making use of the actual and anticipated usage, it's miles some thing but tough to understand the over-burden just as underneath stacked has in CDCs. Mohammad, A.k., et.al., [17], had been expressed strength effective and SLA-mindful dynamic VM union Mechanism (PCM) set of guidelines. in their corporation, they had been considered as severa parameters for approximating the future host usage, as an instance, RAM, CPU and device Bandwidth. They hooked up the competency in their calculation by using using enterprise with different 4 benchmarking calculations via executing energy skillability in CDCs.

\section{THE SYSTEM ARCHITECTURE}

\section{Data Center Model}

In our paintings, the tool is connected on IaaS condition which fuses an considerable range(n) of heterogeneous bodily machines $(\mathrm{PM}=\mathrm{PM} 1, \mathrm{PM} 2, \ldots . . \mathrm{PMn})$. each $\mathrm{PM}$ is definite thru $\mathrm{R}$ type of sources comprehensive of the CPU execution (characterised in MIPS), circle carport capacity, amount of RAM together with gadget facts switch capability. these hosts accommodates of mult-attention CPUs. but, some wide assortment (m) of virtual Machines $(\mathrm{VM}=\mathrm{VM} 1, \mathrm{VM} 2, \ldots . . \mathrm{VMm})$ can be apportioned to every $\mathrm{PM}$ by means of automatic framework discover (VMM) in a few different case called hypervisor. The machine associated garage(NAS) are organized and made do with a application basically based programming software that's

Blue Eyes Intelligence Engineering

\& Sciences Publication 
connected to connotes those PM's adjacent plates. by means of using those characters of host fill the need of stay relocation. The final venture handy of PM and time of VM provisioning is unusual. VM provisioning is requested thru severa individual clients of scattered land regions, and people customers call for for above alluded to parameters of PM. The programming layer includes segments - nearwith the aid of and global directors [18]. neighborhood manager is living as a component of the virtual gadget display (VMM) or hypervisor on every hub of the IaaS engineering. The obligation of near-through director is to acquire the statistics of hub's CPU use through relentless investigating and choosing the $\mathrm{VM}$ via rescaling it for live motion whilst great. so also, it's going to anticipate the reaction time of each VM dependent on the usage of the noteworthy information to discover the old VM that is the parts like the modern VM. concurrently as international manager dwelling within the ace hub and collects the information from nearby directors to put in strength the usage of assist and issuing instructions for the VM scenario enhancement.

\section{Power Model}

Power utilization by a server in server farm is associated with its processor, RAM, hard circle, and data transmission. Late examinations [19] have exemplified that regardless of whether the DVFS approach is applied, the vitality use by servers has a straight association with all out power consumption alongside CPU use. The vitality utilization by a server is developing upward with inactive CPU usage status to completely CPU use status. Subsequently, the server electricity utilization is planned by a straight model capacity of its present CPU utilization (u) as:

$$
\mathrm{P}(u)=\left(\mathrm{P}_{\text {idle }} * \mathrm{P}_{\text {busy }}\right)+\left(1-\mathrm{P}_{\text {idle }}\right) \times u
$$

where, $P$ is the anticipated power-consumption, $\mathrm{P}_{\text {busy }}$ and $P_{\text {idle }}$ are the power-consumption value once a host is at its maximum utilization state and idle state respectively. The CPU's utilization is to be symbolized by $u$. The utilization of a CPU alters time by time. We define it as a function $u(t)$ of time. As a result, overall consumption of energy can be attained by equation 2 :

$$
\text { Energy }=\int_{t} P(u(t)) d t
$$

\section{Methodology}

On this paintings, we are imparting a binpacking based totally definitely method for strength green VM consolidation to make sure electricity performance and plenty much less quantity of VM migration with parameters as power, time in hours, size of VMs and amount of hosts close down the usage of electricity aware Greeny Cloud Dynamic set of regulations(ECGCD). This set of rules is the aggregate of 4 other algorithms together with Predicting the software of a PM - Overload and Underload detection, along side VM choice and VM Allocation algorithms. Migration is the practice to interchange the $\mathrm{VM}(\mathrm{s})$ from one supplyserver to another purpose-server even as the previous is both overloaded, risk-to be overloaded or underloaded. The Migration method includes 3 tiers along side time of migration, nature and amount of VMs to be migrated and nature of the PMs to be placed the aforementioned VMs.

Algorithm 1: Energy Conscious Greeny Cloud Dynamic ( ECGCD) Algorithm
Input: No. of hosts H[];
Output: VMPlacementmap
Interface with cloud data
VM is designed and allocated to hosts;
Workload is assigned to VM;
For each period t;
Identify overloaded, prone-to be overloaded and under loaded host by threshold;
Pick the most suitable VM based on its highest CPU usage;
Consider the least power consumed host and has enough resources from $\mathrm{H} ;$
VM is assigned to the Finest host H[ h];
Place the VM in h;
Return VMPlacementmap

\section{1. TIME OF VM MIGRATION}

Relocation is to be actualized while a PM is both underloaded, over-burden or possibility to be overburden. An edge well worth is applied to differentiate the above scenario.A regular observing is fundamental to understand the weak host machines. any other widespread project is to choose the VMs as in step with the time and conduct to migratefrom the formerly cited feeble hosts with three states.

A. $\quad$ software Prediction of the host
For the heap figuring, we analyze three parameters for instance CPU, records switch capability and memory for the heap computation. every VM has its personal CPU, records transmission and reminiscence. Burden at the VM may be determined as:

$$
\begin{aligned}
v m(c p u)_{\text {usage }} & = \\
\frac{\sum V M_{j}^{\text {mips }}}{\sum P M_{i}^{\text {mips }}} & =
\end{aligned}
$$




$$
\begin{aligned}
& v m(b w)_{\text {usage }}=\frac{\sum V M_{j}^{b p s}}{\sum P M_{i}^{b p s}} \\
& v m(\text { ram })_{\text {usage }}=\frac{\sum V M_{j}^{\text {ram }}}{\sum P M_{i}^{r a m}} \\
& v m_{\text {Util }}=v m(c p u)_{\text {Usage }}+v m(\text { bw })_{\text {Usage }}+v m(\text { ram })_{\text {Usage }}
\end{aligned}
$$

The anticipating of things to come asset prerequisites is conclusive for successful asset execution in CDCs. The vitality use by servers has a direct association with absolute power utilization alongside CPU usage, memory and data transmission. In this work, we are computing vitality utilization of servers dependent on the in advance of referenced different parameters. Yet, Utilization of CPU assumes an essential job in the VM load. So VM burden can be legitimately corresponding to the CPU use of the VM.

$$
V M_{\text {Load }}=V L=\frac{\sum V M_{j}^{\text {mips }}}{\sum P M_{i}^{\text {mips }}}
$$

Total load on the host machine is calculated as the total load of the VM running into that host. If there are $m$ VM on $n^{\text {th }}$ host, then average load on the $\mathrm{n}^{\text {th }}$ host.

$$
P M_{\text {Load }}=\frac{\sum_{j=1}^{m} V L_{j}}{m}
$$

We will not get the accurate host utilization by summing up the CPU, memory and bandwidth directly. Though we are calculating the host utilization based on the compound factors i.e., CPU, RAM and network bandwidth by taking the product to combine them from virtual and physical machines. The host utilization can be calculated by the given equation:

$$
\begin{aligned}
& \boldsymbol{h}(\boldsymbol{u})=\frac{v m_{j}(\mathrm{cpu})_{\text {usage }}}{1-p m(\text { cpu })} * \frac{v m_{j}(\text { ram })_{\text {usage }}}{1-p m(\text { ram })} * \\
& \frac{v_{\boldsymbol{j}}(\boldsymbol{b w})_{\text {usage }}}{1-\boldsymbol{p m}(\boldsymbol{b} w)}, j=1,2, \ldots, m
\end{aligned}
$$

\section{B. Host Overload, Prone-to- overload and Under load Detection Evaluation}

3 element esteems -, for instance, decrease, middle and upper are applied to painting the over-burden, opportunity for over-burdening and underloaded have. the ones features may be Static or Dynamic. attributable to static edge this is pre set and they probably might not be adjusted at some point of the runtime, whilst due to dynamic location, the ones restriction esteems might be altered in the course of the execution time. on this way, dynamic limit is an increasing number of pertinent for the cloud state of affairs, wherein property asked through the
VM are modified powerfully due to non-unsurprising very last burden. VM movement opportunities are raised with those dynamic vicinity esteems. all the more explicitly, the higher the higher part or lesser the decrease restrict should be raised the percentages of VM relocation. In maximum of the work, taken into consideration CPU utilization is surely perspective that is utilized to assess the heap, yet RAM and records transfer capacity are the maximum essential additives in the framework as contrasted and the CPU. therefore, for ascertaining the better limit, on this paper we consider the 3 parameters, as an instance, CPU, RAM and transmission capacity with equal strength.

Upper Threshold Calculation

$$
\begin{aligned}
& T_{c}=\frac{\sum_{j=1}^{m} V M_{j}^{\text {mips }}}{\sum P M_{i}^{\text {mips }}} \\
& T_{r}=\frac{\sum_{j=1}^{m} V M_{j}^{r a m}}{\sum P M_{i}^{\text {ram }}} \\
& T_{b}=\frac{\sum_{j=1}^{m} V M_{j}^{b p s}}{\sum P M_{i}^{b p s}} \\
& T_{t}=\sum\left(T_{c}, T_{r}, T_{b}\right) / 3 \\
& \mathrm{~T}_{\text {up }}=1-\mathrm{x}^{*} \mathrm{~T}_{\mathrm{t}}
\end{aligned}
$$

In which ' $\mathrm{m}$ ' is the whole VM operating within the server and ' $\mathrm{x}$ ' is the level of the server load. It executes as a well being parameter which jam the tradeoff amongst the amount of VM relocation and assets wastages. decrease the estimation of ' $x$ ' will end result the bringing down of the strength usage, yet the higher of it will make the SLA infringement and the opposite way around. by way of the reason of the beyond examinations $\mathrm{x}$ is five $\%$ ie.0.05.Threshold for the following meantime is inspected that is achieved from the recorded data as an example restriction . The $\operatorname{Tr}$ period in-between is relatively relative to the server utilization inside the Tc period in-between.

The forecast of software is achieved right here through utilizing direct relapse method. The capability exhibited the direct affiliation a few of the up and coming and present CPU usage of each single hosts are as following:

$Y=\beta_{0}+\beta_{1} x$

where, $\beta_{0}$ and $\beta_{1}$ are regression coefficient constraints that calculate in accordance with the $r$ previous CPU utilization in a physical host. The $Y$ is the predicted or estimated value of the CPU utilization; $x$ is the predictor which gives current $\mathrm{CPU}$ utilization value.

$$
Y=\beta_{0}+\beta_{1} * \text { currentTotalUtil(16) }
$$



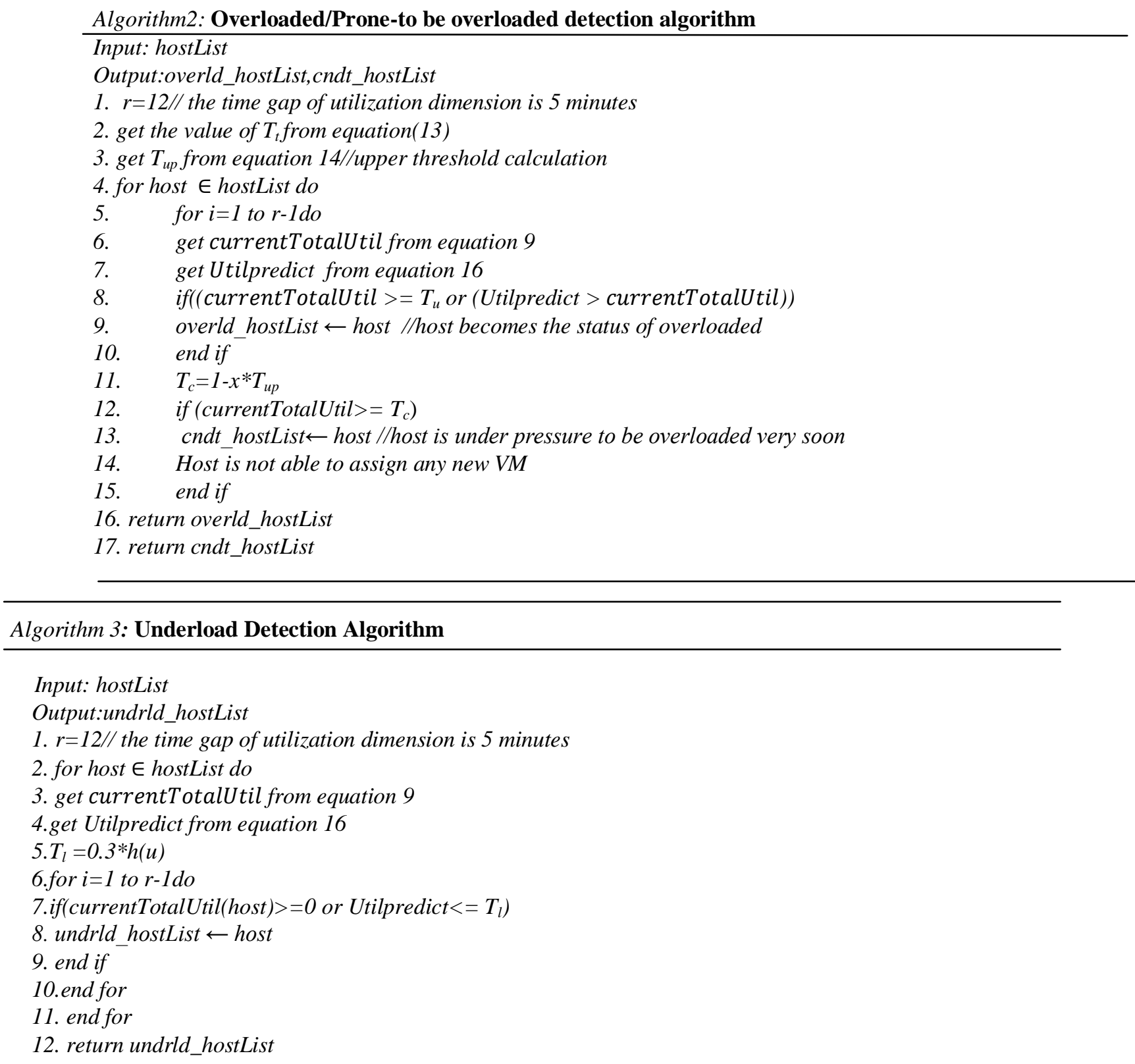

If the CPU utilization of a physical host corresponding of either zero or when the prediction utilization will be less than or equal to $30 \%$ of total host utilization, the host is supposed to be as underloaded host. So as to diminish the energy consumption of that source host it is essential to migrate all VMs to another target host(s). Then the host toggles to the sleep- mode after shifting all VMs. The target host should satisfy three condition while accepting the VMs from ailing hosts:

i. Should not be an overloaded host.

ii. Should not be a candidate for being overloaded.

iii. Should not be an underloaded host.

\section{2. VM SELECTION \& RESULTS}

It's miles primary venture to make a judgment approximately which VM ought to be relocated in light of the fact that it is the first rate check of inflicting all out motion time along down time. Down time $r$ dark out time is a period for which VM isn't always to be had for the cease consumer, where absolute movement time is a period required to shunt the VM to any other server an entirety. within the event that the big expected VM is chosen, the all out relocation time simply as down time could be raised. in the occasion that we pick littlest system, at that factor an sizeable number of VM should be relocated. So in our methodology, we mastermind the $\mathrm{VM}$ in plunging request wherein the dimensions of VMs are greater prominent than or equivalent to the difference a number of the complete host use and upper threshold[19]. 


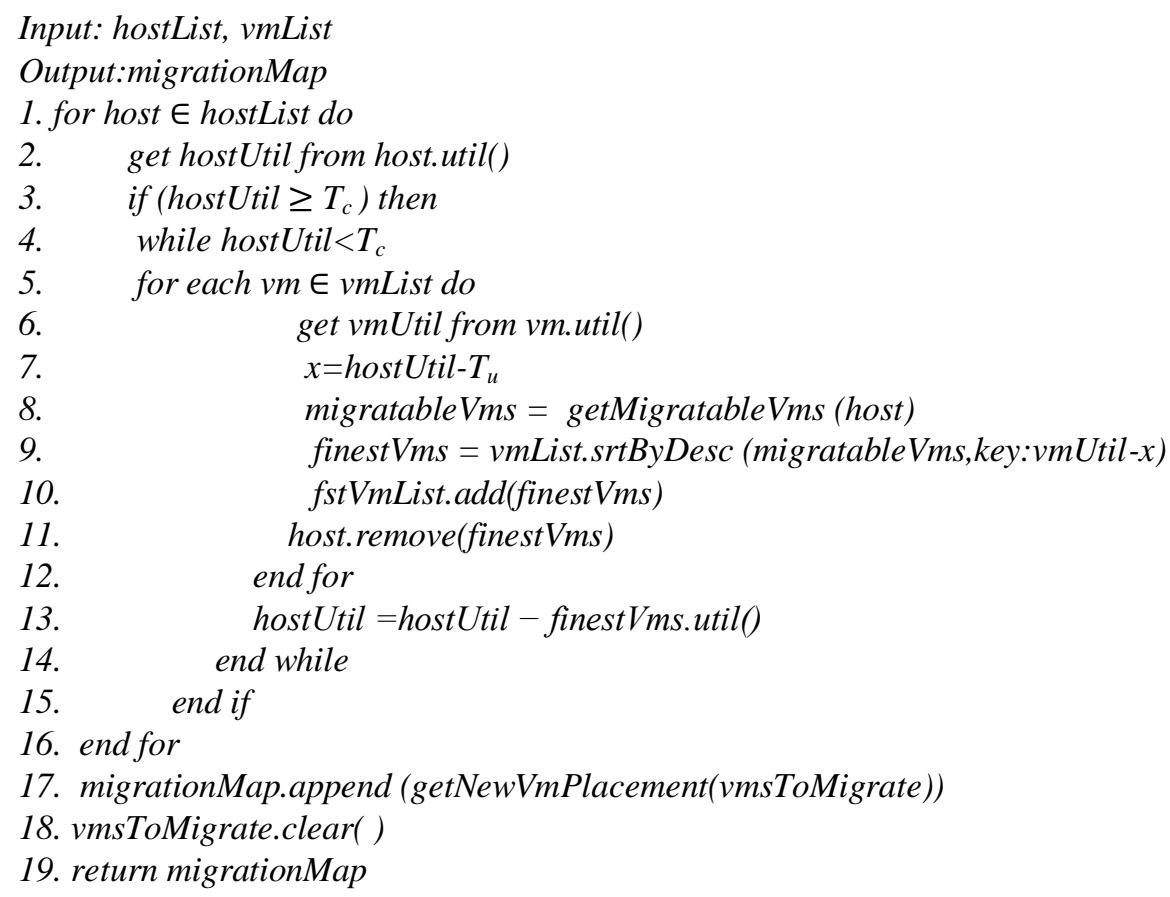

\section{3. HOST SELECTION}

in the course of this level speakme approximately a calculation for VM association. at the factor when a VM is to be picked relocation, it's miles fundamental to discover a reasonable server dependent on VM forte and generally speakme approach well-known in the CDC. ensuing to the VM determination, consequently choose the host wherein the chose VM may be put. The VMP issue might be tested as a canister urgent problem, where receptacles of fluctuating sizes talking to the physical machines and the VMs are considered because the things to be set inside the containers. [20].containers sizes are the cuttingedge CPU limits of the PM and charges are next to the electrical power utilization thru those PMs. it is a NP-tough choice issue in nature. defective willpower of the PM may additionally increase the amount of VM relocation which motive asset wastage and SLA infringement. on this paintings selecting the VM that's energy conscious dependent on the correct restrict estimation of the host simply as least power utilization.

\section{Algorithm 5: PM Allocation Algorithm}

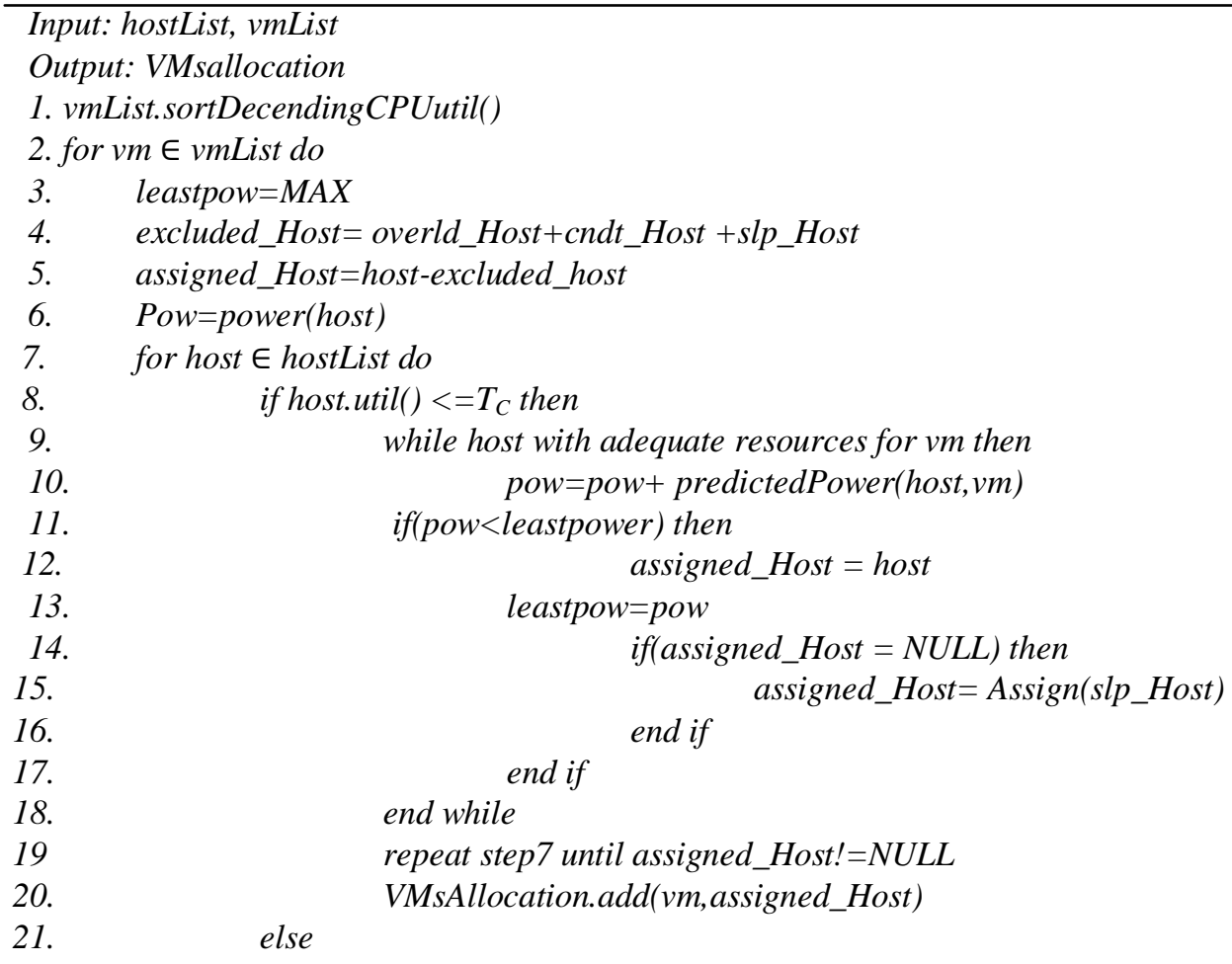


24. end for

25. end for

26. return VMsallocation

\section{Performance appraisal}

\section{a. Workload Data}

To facilitate to evaluate the ECGCD Algorithm, the workload contained data, which are employed for simulation, that are accessed from the CoMon Project, a widely scalable monitoring scheme for PlanetLab in CloudSim [21]. We employ the similar power models supplied in the website for both servers as shown in the following table:

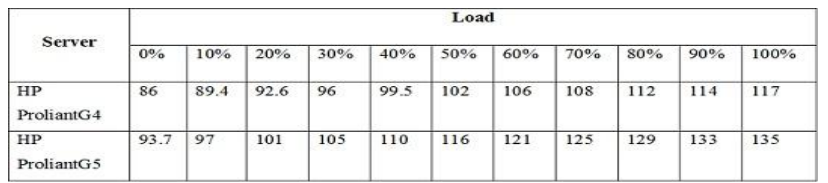

Table 1: The power consumption at diverse load levels in Watts

The features of PMs and VMs used in the research is listing in Table 2 and Table3, respectively.

\begin{tabular}{|l|l|l|}
\hline \multirow{2}{*}{ Parameters } & Server \\
\cline { 2 - 3 } & HP ProliantG4 & HP ProliantG5 \\
\hline No of host & 400 & 400 \\
\hline No of Cores & 2 & 2 \\
\hline MIPS & 1860 & 2660 \\
\hline RAM & 4096 & 4096 \\
\hline BW & $1 \mathrm{~GB}$ & $1 \mathrm{~GB}$ \\
\hline Storage & $1.5 \mathrm{~GB}$ & $2 \mathrm{~GB}$ \\
\hline
\end{tabular}

Table 2: Host Parameters

\begin{tabular}{|l|l|l|l|l|}
\hline \multirow{2}{*}{} & \multicolumn{4}{|l|}{ VM Type } \\
\cline { 2 - 5 } & $\begin{array}{l}\text { High- } \\
\text { CPU } \\
\text { Medium } \\
\text { Instance }\end{array}$ & $\begin{array}{l}\text { Extra } \\
\text { Large } \\
\text { Instance }\end{array}$ & $\begin{array}{l}\text { Small } \\
\text { Instance }\end{array}$ & $\begin{array}{l}\text { Micro } \\
\text { Instance }\end{array}$ \\
\hline $\begin{array}{l}\text { No of } \\
\text { Cores }\end{array}$ & 1 & 1 & 1 & 1 \\
\hline MIPS & 2500 & 2000 & 1000 & 500 \\
\hline RAM & 870 & 1740 & 1740 & 613 \\
\hline BW & $1 \mathrm{MB}$ & $1 \mathrm{MB}$ & $1 \mathrm{MB}$ & $1 \mathrm{MB}$ \\
\hline Storage & $3.85 \mathrm{~GB}$ & $2 \mathrm{~GB}$ & $1.75 \mathrm{~GB}$ & $613 \mathrm{MB}$ \\
\hline
\end{tabular}

Table 3: VM Parameters

The genuine outstanding task at hand follows gives by CoMon Project to CPU usage information of in excess of a 1000 virtual machines from more than 500 physical machines, with the interim of 5 minutes for CPU use for the estimation, from the servers that are arranged everywhere throughout the world. We have haphazardly chosen each VM's outstanding task at hand follows from any of the VMs of 3 days and number of VMs that are studied on every day are appeared in following table:

\begin{tabular}{|l|l|}
\hline $6^{\text {th }}$ March & 1012 \\
\hline $15^{\text {th }}$ March & 1065 \\
\hline $22^{\text {nd }}$ April & 1057 \\
\hline
\end{tabular}

Table 4: The number of VMs in the real workload

\section{a. Performance Metrics}

Energy:

Energy consumption arouse by a CDC counts the whole amount of energy used by various apparatus of the CDC such as CPU, storage, RAM, energy supply chambers and air cooling organisms. The overall energy utilization can be attained by equation 5.9:

$$
\text { Energy }=\int_{t} P(u(t)) d t
$$

Time in hours:

The real extraordinary undertaking to hand follows gives thru CoMon mission to CPU utilization statistics of in more of a one thousand digital machines from more than 500 physical machines, with the intervening time of five mins in 24 hours of three arbitrary days for CPU use for the estimation, from the servers which is probably organized everywhere in the course of the world. size of the VM:

The size of the VMs suggests the CPU utilization of the $\mathrm{VM}(\mathrm{s})$ which might be greater noteworthy than or identical to the range in the middle of the whole host use and higher limit esteem.

\section{Type of VM Migration:}

This measurement indicates the recurrence of VM relocation finished in $\mathrm{PM}(\mathrm{s})$. inside the event that regularly wide variety of VMs are relocated this may be the purpose for execution corruption and a key aspect for SLA infringement. the alternative way around, if there may be a pair VM movements, that could direct to an irrelevant venture. in spite of the truth that an adjusting is essential while thinking about this example.

extensive sort of PM shutdown:

This presentation metric determines that how lots of the time the PMs are changing to shutdown. The better measure of reactivation of the PM may also additionally boom the power utilization of the CDCs. So it is important to shutdown the inactive PMs while it's far recognized to be underloaded. the continued replacing of the PM with ON and off kingdom may be the cause for PM breakdown on the long run. So it ought to be directed. 


\section{A Heuristic Research Of The Designing And Evaluation Of Energy Efficiency Using Dynamic Vm Consolidation In Green Cloud Data Centres}

\section{c. $\quad$ Experimental Setup}

we've proposed a big scale IaaS circumstance which offer considerable processing property to its clients. For the evaluation the adequacy of our check, executions were acting on the CloudSim duplicate toolbox. within the allocated computing network, CloudSim is polishing off constantly regularly properly referred to as a end end result in their assist for flexible, adaptable, capable and rehashed examination of asset imparting techniques for one in all a kind programs [22]. The proposed calculation ECGCD is assessed with energy conscious asset challenge calculations HCLB[23] and MeReg[24].

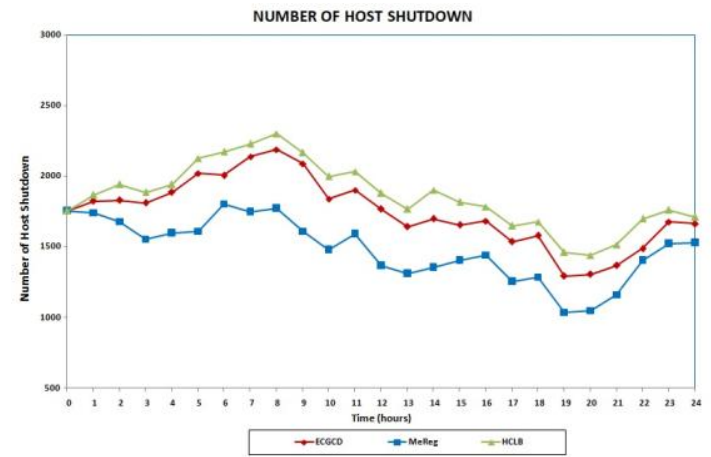

Fig. 1: Number Host Shutdown by ECGCD and other energy aware resource allocation algorithms for random workload traces(mean values)

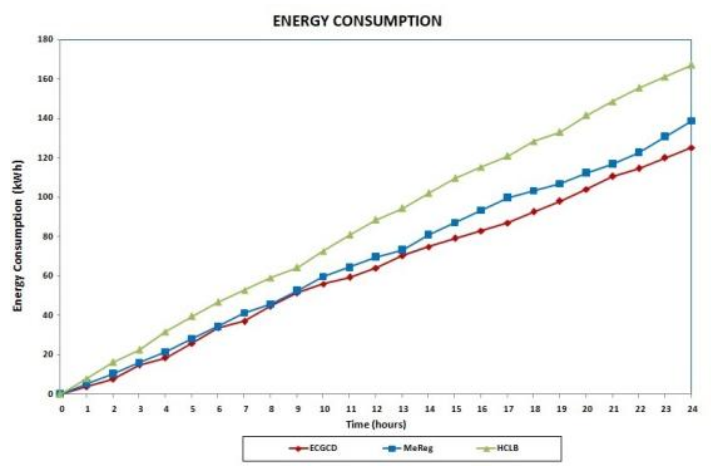

Fig. 2: Energy consumption by ECGCD and other energy aware resource allocation algorithms for random workload traces(mean values)

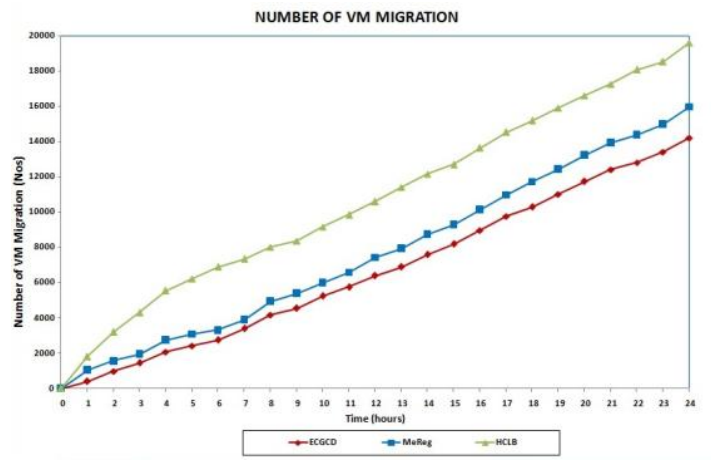

Fig. 2: VM migration by ECGCD and other energy aware resource allocation algorithms for random workload traces(mean values)
This proposed set of on-line ECGCD displaying the mild charge of the host shutdown in Fig.1 as compared with specific existing algorithms consisting of HCLB and MeReg. every the PM shutdown boom or decrease is probably the purpose of large power intake which explained in performance matrics segment.

The least electricity consumption is as compared with modern-day strength conscious algorithms as proven in Fig. 2. The algorithm ECGCD, gaining access to power saving round $33.47 \%$ and $10.78 \%$ as weighed against HCLB and MeReg correspondingly because the advocate value of three random date values respectively. furthermore, the variety of migration is also reduced through $38.04 \%$ and $12.32 \%$ even as evaluating with the aforementioned power conscious algorithms HCLB and MeReg respectively (Fig. 2). This achievement can be received due to the findings of dynamic threshold values are aided for converting idle servers to low-energy nation (i.e., sleep or hibernation) to obtain the least intake of strength.

\section{CONCLUSION}

Dynamic consolidation of VMs is a powerful method to broaden the assets utilization as well as power performance in CDCs. This technique plays an essential function of plummeting strength consumption which might carry down the $\mathrm{CO} 2$ emission and accelerate ROI in favour of cloud provider vendors via turning off the inactive host machines or positioned the machines in lowstrength mode. in this paper we've furnished energy aware Dynamic VM Consolidation with 3 computerized adjustment of threshold cost including top , middle and reduce threshold to discover the overloaded or at risk of be overloaded or underloaded host tool. we have appraised the proposed algorithm for cloud's big-scale IaaS surroundings thru vast simulation on CloudSim 3.zero toolkit. PlanetLab workload of CloudSim toolkit is used for the simulation. It need to be opted as a simulation platform due to the fact as it offers all the services of Cloud computing structure. we've got tailored the simulator itself to carry out our studies art work in Cloud platform. To ensure the competence of our technique we compare ECGCD, the proposed one with distinct modern energy conscious beneficial resource allocation set of rules along with HCLB and MeReg. The proposed technique verifies the development of the CDC sources utilization and diminishes strength intake, on the identical time as assuring the characterized QoS constraints.

\section{REFERENCES}

1. Namasudra, S. (2018). CLOUD COMPUTING: a new generation. magazine of essential and accomplished Sciences, 10(2), 113-a hundred thirty 5.

2. Garcia, P. A., Fernández, J. M. M., Rodrigo, J. L. 
A., \& Buyya, R. (2017). Proactive energy and Thermal conscious Optimizations for energy-efficient Cloud Computing.

3. EC-ecu commission. (2007). restricting international weather trade to 2 tiers Celsius. The manner in advance for 2020 and beyond. COM/2007/2. (Accessed in December, 2018).

4. Murugesan, S., and Gangadharan, G. R. (2012). Harnessing green IT: concepts and practices. Wiley Publishing. ISBN: 978-1-119-97005-7.

5. Ferdaus, M. H., Murshed, M., Calheiros, R. N., \& Buyya, R. (2017). Multi-aim, Decentralized Dynamic digital machine Consolidation the usage of ACO Metaheuristic in Computing Clouds. arXiv preprint arXiv:1706.06646.

6. Abali, B., Canturk I., Jeffrey O. ok., Suzanne k. M., and Dipankar S.(2017) "stay digital system migration 86f68e4d402306ad3cd330d005134dac of service." U.S. Patent utility $15 / 448,268$.

7. Abdullah, M., Lu, good enough., Wieder, P., and Yahyapour, R. (2017). A Heuristic-based approach for Dynamic VMs Consolidation in Cloud information facilities. Arabian magazine for technological bdd5b54adb3c84011c7516ef3ab47e54 and Engineering, 115.

8. Nathuji, R., \& Schwan, ok. (2007, October). Virtualpower: coordinated energy manage in virtualized organization structures. In ACM SIGOPS running systems overview (Vol. 41, No. 6, pp. 265-278). ACM.

9. Esfandiarpoor, S., Pahlavan, A., \& Goudarzi, M. (2015). form-aware 8db290b6e1544acaffefb5f58daa9d83 virtual device consolidation for datacenter strength development in cloud computing. computer systems \& electric powered Engineering, forty, seventy 4-89.

10. Quanwang, W., Fuyuki, I., Qingsheng, Z., and Yunni, $X .(2016)$ "strength and migration price-aware dynamic virtual device consolidation in heterogeneous cloud datacenters", IEEE Transactions on offerings Computing, volume: PP, problem: ninety nine.

11. Fahimeh, F, Adnan,A., Tapio ,P., Pasi, L. ,Juha P., Ivan P.,and Hannu T.,(2015) "using Ant Colony machine to Consolidate VMs for inexperienced Cloud Computing",IEEE Transactions On offerings Computing, Vol. eight, NO. 2, .

12. Ibrahim A., Karim Djemame, Django Armstrong and Richard Kavanagh, "electricity- aware profiling for Cloud Computing Environments", digital Notes in Theoretical pc technological bdd5b54adb3c84011c7516ef3ab47e54 in Article volume 318, pp. 91-108 • Elsevier November2015

13. Youwei, D., Xiaolin, Q., Liang, L., and Taochun,W.(2015)“electricity green scheduling of virtual machines in cloud with on linecut-off date constraint", destiny era pc structures, ElsevierVolume 50, trouble C, Pages sixty two-seventy four .

14. Zhe, H and Danny, H.k.T.(2016) "M-convex VM Consolidation: towards a higher VM Workload Consolidation", IEEE Transactions on Cloud Computing, quantity: four,hassle: four.

15. Mustafa, S., Bilal, ok., Malik, S. U. R., \& Madani, S. A. (2018). SLA-aware electricity green useful resource control for Cloud Environments. IEEE get admission to, 6, 15004-15020.

16. Nguyen, T. H., Di Francesco, M., and Yla-Jaaski, A. (2017). virtual device Consolidation with more than one utilization Prediction for electricity-efficient Cloud information centers. IEEE Transactions on offerings Computing.

17. Mohammad, A.adequate., Mohd, N.D., Azizol, A., Shamala, S., and Mohamed, O.(2017) "strength-green Algorithms for Dynamic virtual gadget Consolidation in Cloud data centers", IEEE Transactions on inexperienced Cloud and Fog Computing: energy efficient and
Sustainable Infrastructures, Protocols and packages Vol.five , trouble: 69 PP no: 10709 - 10722.

18. Beloglazov, A., and Buyya, R. (2013). dealing with overloaded hosts for dynamic consolidation of digital machines in cloud statistics facilities below $86 f 68 \mathrm{e} 4 \mathrm{~d} 402306 \mathrm{ad} 3 \mathrm{~cd} 330 \mathrm{~d} 005134 \mathrm{dac}$ of carrier constraints. IEEE Transactions on Parallel and distributed systems, 24(7), 1366-1379.

19. Gutierrez-Garcia, J. O., and Ramirez-Nafarrate, A. (2015) Collaborative marketers for dispensed load management in cloud statistics centers the usage of stay migration of virtual machines. IEEE transactions on services computing, eight(6), 916-929.

20. F. Farahnakian, T. Pahikkala, P.Liljeberg,J.Plosila, N.TrungHieu, and H.Tenhunen, (2016)"strength-conscious VM Consolidation in Cloud records facilities the use of utilization Prediction version", IEEE Transactions on Cloud Computing quantity: PP, trouble: ninety nine.

21. Dan, good enough., Sasa, M., Matej, G., and Ondrej, P.(2017)"testing internetapplications and offerings the use of PlanetLab", quantity 53, Pages 33-38.

22. Rodrigo, N. C., Rajiv, R.,Anton, B., C'esar, A. F. De Rose and Rajkumar, B.(2015) "CloudSim: A toolkit for modeling and simulation of cloud computing environments and assessment of useful resource provisioning algorithms", software: exercise and experience, vol. forty one, no. 1, pp.23-50. [23]

23. Pantazoglou, M., Tzortzakis, G., and Delis, A. (2016). Decentralized and power-inexperienced workload management in corporation clouds. IEEE transactions on cloud computing, four(2), 196-209.

24. Yadav, R., and Zhang, W. (2017). MeReg: managing electricity-SLA Tradeoff for green cellular Cloud Computing. wi-fi Communications and mobile Computing, 2017. 